\title{
Minority stress and safer sex practices among sexual minority women in Toronto, Canada: Results from a cross-sectional Internet-based survey
}

Logie, Carmen H.; Lacombe-Duncan, Ashley; MacKenzie, Rachel K.; and Poteat, Tonia

Version Post-Print/ Accepted Manuscript

Citation Logie, Carmen H., Lacombe-Duncan, Ashley, MacKenzie, Rachel K., (published version) and Poteat, Tonia. (2016). Minority stress and safer sex practices among sexual minority women in Toronto, Canada: Results from a crosssectional Internet-based survey. LGBT Health 3(6): 407-415. doi: 10.1089/lgbt.2016.0005.

Publisher's Statement Final publication is available from Mary Ann Liebert, Inc., publishers http://dx.doi.org/10.1089/lgbt.2016.0005.

Always cite the published version, so the author(s) will receive recognition through services that track citation counts, e.g. Scopus. If you need to cite the page number of the TSpace version (original manuscript or accepted manuscript) because you cannot access the published version, then cite the TSpace version in addition to the published version using the permanent URI (handle) found on the record page. 
12 Corresponding Author:

13 Carmen H. Logie, PhD

14 Factor-Inwentash Faculty of Social Work, University of Toronto

15246 Bloor Street West

16 Toronto, Ontario, Canada

17 M5S 1V4

18 Tel: $1(647) 4544203$

19 Fax: 1 (416) 9787072

20 carmen.logie@utoronto.ca

21

22

23

24

25

26

27

28

Co-authors email addresses:

Ashley Lacombe-Duncan, M.S.W. (ashley.lacombe.duncan@utoronto.ca)

Rachel K. MacKenzie, M.P.H. (rk.mackenzie@mail.utoronto.ca)

Tonia Poteat, Ph.D. (tpoteat@jhu.edu)

Running head: Minority stress and safer sex for SMW

\section{Results from a cross-sectional internet-based survey}

Tonia Poteat, $\mathrm{PhD}^{4}$

${ }^{1}$ Factor-Inwentash Faculty of Social Work, University of Toronto, Toronto, Ontario, Canada.

${ }^{2}$ Women's College Research Institute, Women's College Hospital, Toronto, Ontario, Canada,

${ }^{3}$ Dalla Lana School of Public Health, University of Toronto, Toronto, Ontario, Canada.

${ }^{4}$ Johns Hopkins Bloomberg School of Public Health, Johns Hopkins University, Baltimore, MD, 


\section{Abstract}

Purpose: Sexual stigma is a chronic stressor that enhances vulnerability to mental health disparities among lesbian, gay, bisexual, and queer people. Sexual stigma has also been associated with reduced uptake of safer sex practices, such as condom use, among gay and bisexual men. Scant research has examined the relationship between sexual stigma and safer sex practices among sexual minority women (SMW), including lesbian, bisexual and queer women.

Methods: We explored associations between sexual stigma and safer sex practices among SMW. We also tested the interaction between sexual stigma, social support, and resilient coping in this relationship. A cross-sectional internet-based survey was administered to SMW in Toronto, Canada.

Results: Among 388 participants with complete measurement data, simple linear regression indicated both perceived and enacted sexual stigma were positively associated with uptake of safer sex practices. In multivariable analyses, significant interactions were found between perceived sexual stigma and resilient coping, and between enacted sexual stigma and social support. At low levels of resilient coping, higher levels of perceived sexual stigma were associated with with fewer safer sex practices, while at high levels of resilient coping the relationship was reversed. At low levels of social support, higher levels of enacted sexual stigma were associated with fewer safer sex practices, while at high levels of social support the relationship was reversed.

Conclusions: These findings document complex relationships between sexual stigma dimensions, coping, social support, and safer sex practices. Understanding the role these variables play in uptake of safer sex practices can inform sexual health interventions tailored for SMW.. 
52

53

54 Keywords: discrimination, minority stress, prevention, sexual minority women, sexual stigma, 55 STD

56 


\section{Introduction}

Approximately one-fifth of lesbian, bisexual, queer (LBQ) and other sexual minority women (SMW) - a diverse community of women attracted to other women, people of multiple genders, transgender, and gender fluid persons - have a lifetime history of sexually transmitted infections (STIs), similar to STI infection rates among heterosexual women. ${ }^{1-4}$ Yet perceptions that SMW are at low risk for STI and HIV acquisition persist and contribute to a paucity of research on safer sex practices among SMW., ${ }^{2,5-8}$

The limited research examining sexual practices among SMW has revealed inconsistent uptake of safer sex strategies. ${ }^{9,10}$ For example, a multi-country study with SMW reported low barrier use $(<25 \%)$ among participants. ${ }^{10}$ There is limited understanding, however, of the type and frequency of safer sex strategies used by SMW or factors associated with uptake of safer sex practices. This information could guide intervention development to increase uptake of safer sex practices among SMW. Stigma and discrimination may constrain or enable safer sex practices. ${ }^{11,12}$ Understanding associations between stigma and discrimination and safer sex practices among SMW can inform tailored STI prevention strategies for this overlooked population.

\section{Theoretical approach}

Sexual stigma refers to social and institutional processes and structures that devalue and limit access to power and opportunities among lesbian, gay, bisexual, transgender, and queer (LGBTQ) people and communities. ${ }^{13,14}$ Meyer's minority stress model ${ }^{14,15}$ conceptualizes sociocognitive processes by which sexual stigma experienced by LGBTQ people is a chronic, cumulative stressor that negatively affects health and wellbeing. The minority stress model includes distal processes, such as enacted stigma, whereby people experience acts of violence and unequal treatment (e.g. harassment), and proximal processes, such as perceived (also 
81 referred to as felt) stigma, whereby people experience concerns of rejection and negative

82 treatment by others because of actual or perceived LGBTQ identity, and internalized stigma,

83 whereby people may experience feelings of shame and self-blame. ${ }^{13-16}$

84 The stress of sexual stigma contributes to mental health disparities among LGBTQ

85 populations. ${ }^{14,17,18}$ Conceptualizations of distal (enacted sexual stigma) and proximal (perceived

86 sexual stigma) stressors have been applied to understand mental health outcomes, including

87 suicidality and substance use, ${ }^{19,20}$ across various contexts. ${ }^{21}$ There is growing attention to sexual

88 stigma as a structural driver of HIV disparities. ${ }^{11,22}$ Stigma may influence both individual and

89 group level HIV and STI vulnerability by reducing uptake of safer sex practices (e.g. condom

90 use) and access to sexual health services (e.g. STI testing). ${ }^{12,13}$ A meta-analysis of 16 studies

91 reported correlations between internalized stigma and sexual risk practices among gay, bisexual,

92 and other men who have sex with men (MSM). ${ }^{23}$ Peer victimization, a form of enacted sexual

93 stigma targeting LGBTQ youth, has been associated with sexual risk practices among grouped

94 samples of young LGBTQ youth of diverse genders and sexual orientations. ${ }^{24,25}$ Few studies

95 have explored the relationship between perceived sexual stigma and sexual risk practices.

96 However, one study that pooled over 3000 MSM respondents from 115 countries reported that

97 perceived sexual stigma was associated with significantly reduced access to free condoms, free

98 lubricants, and HIV testing. ${ }^{26}$

Coping and social support have long been recognized as resources that may affect the

100 association between stress-initiated psychological processes such as stigma and poor mental

101 health. ${ }^{27}$ Meyer's minority stress model ${ }^{14}$ posited that coping and social support could moderate

102 the influence of minority stress stigma on mental health outcomes. A sense of belonging to 
103 LGBTQ communities might foster support and solidarity that could contribute to developing 104 adaptive coping mechanisms such as resilience. ${ }^{14}$

105 While social support and stigma may moderate the associations between sexual stigma 106 and mental health outcomes, ${ }^{14,17}$ few studies have explored the relationship between social 107 support and coping and sexual risk practices among LGBTQ populations. Some evidence 108 suggests that maladaptive coping styles effect sexual risk among MSM. Yi et al. ${ }^{28}$ found that 109 disengagement coping fully mediated the association between internalized sexual stigma and 110 condomless anal intercourse among a U.S. sample of MSM. Literature examining social support, 111 stigma, and sexual risk practices is limited and suggests that social support does not affect sexual 112 risk practices. ${ }^{29} \mathrm{We}$ found no studies that examined coping style or social support and their 113 associations with safer sex practices among SMW. However, one study ${ }^{30}$ conducted among 114 'mostly heterosexual' compared to 'exclusively heterosexual' women found that social support 115 partially mediated the relationship between sexual orientation and sexual risk practices, defined 116 as age at first intercourse, ever having had an STI, or lifetime number of sexual partners. In summary, knowledge gaps remain regarding enacted and perceived sexual stigma and 118 sexual risk practices among SMW. In addition, little is known about protective factors — such as 119 coping strategies and social support — and their association with safer sex practices among SMW.

120 Our study sought to address these gaps by assessing: a) the frequency and types of safer sex 121 practices used; b) associations between sexual stigma and uptake of safer sex practices; c) 122 interactions between social support and resilient coping with uptake of safer sex practices, 123 among SMW in an urban Canadian setting. Hypotheses included: 1) higher levels of sexual 124 stigma (perceived, enacted) would be associated with lower levels of safer sex practices; 2) 
125 social support and resilient coping would moderate the associations between sexual stigma and 126 safer sex practices.

\section{Materials and Methods}

128

Sampling and recruitment

Between December 2011 and January 2012, we conducted a cross-sectional internetbased survey with SMW in Canada. Inclusion criteria included: adults over 18 years old; selfidentified lesbian, bisexual, gay, queer, or other self-defined sexual orientation, and/or same sex attracted self-identified women; and living in the Greater Toronto Area. We refer to sexual minority women (SMW) in this study; although used in research as an umbrella term for women of multiple sexualities we recognize its limitations in reflecting elements of culture and identity. ${ }^{31}$

Ten peer recruiters (PRs) were purposively selected based on their relationship with the LGBTQ community as service providers, community organizers, and event planners/promoters, as well as self-identification as SMW with diverse sexual identities, ages and ethnicities with the aim of reaching a broad range of SMW. Using a modified peer-driven recruitment strategy, ${ }^{32}$ each PR aimed to recruit a minimum of 25 participants using a combination of strategies (e.g., in-person, internet). Convenience sampling techniques, including sending emails regarding the study to LGBTQ agencies, community health centers, HIV/STI service organizations, and ethnocultural agencies, were utilized to enhance recruitment.

The survey was pilot tested in a focus group with PRs, then modified to enhance clarity and appropriateness. Subsequently, participants completed the self-administered 45-60 minute online survey in a location of their choice. Participants could opt to receive a \$20 gift card honorarium. Research ethics approval was granted from Women's College Hospital, Toronto, 
148 Canada. Prior to completing the survey, participants were presented with an online informed

149 consent form. Upon checking 'yes' to understanding and providing informed consent, the survey 150 opened. For those participants who chose 'no', the survey was terminated.

$151 \quad$ Measures

The survey assessed socio-demographic information, safer sex practices, and potential 153 individual and structural correlates of safer sex practices. Socio-demographic information 154 including age, ethnicity, education, income, relationship status, gender and gender identity, and 155 gender of sexual partners, was collected. Self-identified sexual orientation was collected using a 156 question with the following mutually exclusive options: lesbian, bisexual, gay, queer, 157 heterosexual, and other sexualities.

The primary outcome of safer sex practices was measured using the 'Safer Sexual

159 Practices among Lesbian Women' scale ${ }^{33}$ (Cronbach's $\left.\alpha=0.67\right)$. One item on bondage, 160 dominance, and sadomasochism was removed per PR feedback in pilot-testing. Items were 161 summed, with a higher score indicating higher utilization of safer sex practices. Individual items 162 in this measure were also used to assess the frequency of specific safer sex practices. Sexual stigma was assessed using the Homophobia Scale, ${ }^{34}$ adapted for SMW. ${ }^{35}$ The 164 perceived sexual stigma sub-scale (possible range: $0-20)$ consisted of 5 summed items (e.g. 165 'How often have you felt your family was hurt and embarrassed because you are lesbian, 166 bisexual or queer?') and the enacted sexual stigma sub-scale (possible range 0-28) consisted of 7 167 summed items (e.g. 'How often have you been hit or beaten up for being lesbian, bisexual or 168 queer?'). Sub-scale reliability was acceptable: perceived sexual stigma sub-scale, (Cronbach's $169 \alpha=0.70)$, enacted sexual stigma sub-scale, (Cronbach's $\alpha=0.72$ ), and overall sexual stigma scale 170 (Cronbach's $\alpha=0.78$ ). 
Healthcare utilization was assessed by asking whether the participant had undergone an

172 HIV test, STI test, mammogram, Pap smear, or breast exam in the past 2 years. The answers to

173 these 5 items were summed to form a Health Care Utilization score, with each test adding one

174 point to the overall score. A higher score indicated greater use of healthcare services (Cronbach's

$175 \alpha=0.69)$. Brief resilient coping was measured using Sinclair et al.' ${ }^{36}$ Brief Resilient Coping scale

176 (Cronbach's $\alpha=0.69)$ (range: 4-20). This scale consists of 4 items (e.g. 'I believe that I can grow

177 in positive ways by dealing with difficult situations'). ${ }^{36}$ A higher score indicates higher resilient

178 coping, reflecting belief in one's ability to overcome adverse circumstances. ${ }^{36}$ Social support was

179 assessed using the Multidimensional Scale of Perceived Social Support, a 12- item questionnaire

180 (Cronbach's $\alpha=0.91$ ) that assesses support from family, friends, and a significant other ${ }^{37}$ (range:

181 12-60). The scale includes items such as 'I can talk about my problems with my friends', with a

182 higher score indicating a higher amount of perceived social support from multiple sources.

183 Statistical analysis

All statistical analysis was performed in Stata $14 .{ }^{38}$ We conducted descriptive analyses of socio-demographic variables as well as the outcome and exposure variables, utilizing means and

186 standard deviations for continuous variables and frequencies and proportions for categorical

187 variables. There were 466 women who participated in the internet-based survey. The dataset for

188 this analysis was restricted to the 388 participants who responded to all questions on the Safer

189 Sexual Practices among Lesbian Women scale. Socio-demographic characteristics of included

190 participants were compared to those not included using a Welch two-sample t-test for continuous

191 variables and Fisher's exact test for categorical variables.

In the analytic sample, the distributions of individual safer sex practices were

193 summarized overall and by gender of sexual partners; chi-squared tests were used to compare the 
194 distribution by different categorical variables. Simple linear regression was used to assess the

195 unadjusted association of perceived and enacted sexual stigma, as well as socio-demographic

196 characteristics and social support and resilient coping with an individuals' safer sex practices

197 score.

A multivariable linear regression model was fit to the data based on the hypothesized

199 relationship between minority stress and safer sex practices. This model posited that both

200 perceived and enacted sexual stigma would be associated with reduced safer sex practices, and

201 that social support and resilient coping would reduce the strength of the negative associations

202 between sexual stigma and safer sex practices. Reported history of a male partner, current

203 relationship status, and healthcare utilization were included as potential confounders and

204 therefore control variables. All variables were kept in the model and statistical significance of

205 interactions were tested using the likelihood ratio test.

\section{Results}

207 Participant socio-demographic characteristics

Participant sociodemographic characteristics are summarized in Table 1. Participants

209 ranged in age from 18 to 70 , with a mean age of 31.2 (SD: 8.0). The majority of participants

210 identified as either queer (45.5\%) or lesbian (30.1\%). A total of 121 women, including

211 participants from each category of self-identified sexual orientation, reported a history of at least

212 one male sex partner. Sexual practices and main outcome variables are presented in Table 2.

213 Participants reported a mean of 20.4 (SD 32.7) lifetime sexual partners. Approximately one-fifth

$214(21.8 \%)$ of participants reported either living with an STI currently or having an STI in the past.

215 Participants included in the analysis did not differ significantly in socio-demographic

216 characteristics or main outcomes scores from participants excluded from analyses (not shown). 
Ninety-one percent of respondents reported at least one instance of enacted sexual stigma

218

219

220

221

222

223

224

225

226

227

228

229

230

231

232

233

234

235

236

237

238

239

and over $99 \%$ of respondents reported at least one instance of perceived sexual stigma. Mean

scores for enacted and perceived sexual stigma were 3.5 (SD 2.7) and 8.4 (SD 3.5), respectively.

Uptake of safer sex practices

A summary of uptake of specific safer sex practices for women who did and did not

report having male partners is presented in Table 3 . The uptake of individual safer sex practices

by relationship status is presented in Table 4 . Women in polyamorous relationships or with

multiple partners reported the highest uptake (always/sometimes use) of safer sex strategies for half of the items, with the exception of use of dental dams, use of saran wrap, cutting condoms open for oral sex and not having sex during her/her partners' menstruation.

Associations between sexual stigma and safer sex practices

The unadjusted relationships between variables and safe sex scores can be seen in Table 5. Simple linear regression revealed that contrary to our hypotheses, perceived and enacted sexual stigma were each positively associated with uptake of safer sex practices $(p<0.01)$. For example, an 8-point increase on the perceived sexual stigma sub-scale or a 6-point increase on the enacted sexual stigma sub-scale were each associated with an average of approximately one additional safe sex practice.

The results of the multivariable model exploring the relationship between sexual stigma and safer sex practices while controlling for potential confounding factors can be seen in Table 6 . In multivariable modeling, no significant interactions were found between enacted sexual stigma and resilient coping, nor between perceived sexual stigma and social support. However, significant interactions were found between enacted sexual stigma and social support as well as between perceived sexual stigma and resilient coping. Social support and resilient coping were 
240

241

242

243

244

245

246

247 248

249

250

251

252 253 SMW.

maintained as continuous variables for the multivariable modeling. To demonstrate the interactions, the associations between enacted and perceived stigma at 3 different levels of social support or resilient coping, respectively, have been graphically depicted in Figures 1 and 2. At lower levels of resilient coping, increasing levels of perceived sexual stigma were associated with fewer safer sex practices while at higher levels of resilient coping, the relationship was reversed (Figure 1). Similarly, at lower levels of social support, increasing levels of enacted sexual stigma were associated with fewer safer sex practices while at higher levels of social support, the direction of association was reversed (Figure 2).

\section{Discussion}

Our study is one of the first to apply the minority stress model to better understand uptake of safer sex practices among SMW. We identified complex relationships between dimensions of sexual stigma (enacted, perceived), resilient coping, social support and safer sex practices that suggest the utility of the minority stress model for understanding safer sex practices among

Our findings are congruent with the minority stress model that posits that coping and social support can moderate the relationship between stressors targeting LGBT people and health outcomes. ${ }^{14}$ We found that levels of coping and social support significantly influenced the relationship between sexual stigma and safer sex practices. Participants with lower adaptive coping levels reported reduced safer sex practices when experiencing perceived stigma. This finding builds on prior studies with MSM that demonstrated maladaptive coping mediated the relationship between internalized sexual stigma and condomless sex among MSM. ${ }^{28}$ It is possible that higher resilient coping — positive adaptations to stress ${ }^{36}$ — could play a larger role in moderating the effect of perceived stigma, in comparison with enacted stigma, because perceived 
263 stigma is rooted in perceptions and fears of mistreatment. These perceptions may be more 264 amenable to the intrapersonal processes in resilient coping than experiences of physical or verbal 265 harassment experienced in enacted stigma. Disentangling how coping styles may mitigate 266 dimensions of sexual stigma warrants further investigation. Social support level also resulted in differences in safer sex practices. It is plausible that

268 high levels of support from family, friends, and a significant other could lead to support, 269 solidarity, and community connectedness when someone experiences enacted stigma. ${ }^{39}$ Future 270 research should explore the role of supportive ties in mitigating impacts of stressors such as 271 sexual stigma on safer sex practices.

273 reverses rather than disappears with higher levels of coping/social support. This suggests a type 274 of resilience borne from having social support or coping skills that "turn up" when exposed to 275 sexual stigma, such that one is more likely to practice safer sex. This seems consistent with 276 literature that suggests manageable stress levels may not be harmful, and in fact can promote 277 resilience, whereas chronic, higher levels of stress cause psychobiological harms. ${ }^{40}$ There is a 278 need for additional research on psychobiological effects of different types and levels of sexual 279 stigma.

280 Similarly to Schick et al., ${ }^{10}$ we found that under half of participants responded that they 281 consistently do not share sex toys. Richters et al. ${ }^{41}$ investigated SMW's dental dams usage during $282 \operatorname{sex}(n=543)$ and found approximately $10 \%$ had used a dental dam during oral sex in the previous 2835 months and $2 \%$ had used one often. ${ }^{41}$ Our findings are consistent in that $2 \%$ of women report 284 always using a dental dam. Our study also highlights that most SMW know about, but do not 285 use, barriers, during sex. Future qualitative research may explore barriers to practising safer sex 
286 and strategies to enhance uptake of safer sex practices. Another potential explanation for 287 associations between sexual stigma and safer sex practices is that individuals with increased 288 stigma may have fear or discomfort regarding same sex sexual contact; this is an area for future 289 research as well.

Our study also highlights the role of relationship status and safer sex uptake among

291 SMW. Richters et al. ${ }^{41}$ found that dental dam use was lower among women with more sexual

292 partners, or women who had casual or group sex. Contrary to these findings, we found that 293 women in polyamorous relationships or with multiple partners had the highest uptake of safer 294 sex strategies, with married women reporting the least. Schick et al. ${ }^{10}$ also reported that women 295 not in a relationship were more likely to report using a condom on a shared dildo. Further 296 research is needed to understand the role that relationship type plays in practising safer sex. Uptake of safer sex practices was also correlated with increased healthcare utilization, 298 suggesting that routine primary care check-ups may provide an opportunity for healthcare 299 providers to discuss safer sex practices among SMW. ${ }^{42}$ From a practice perspective, our findings also suggest that interventions to address 301 resilient coping and social support for SMW may mitigate the effect of sexual stigma on sexual 302 risk practices. There is a dearth of interventions to address safer sex strategies among 303 SMW. ${ }^{2,5,6,43}$ A group-based STI prevention intervention with SMW in Canada reported a 304 sustained decrease in sexual risk practices and sexual stigma, yet was not able to effect sustained 305 changes in resilient coping or social support. ${ }^{44}$ Intervention research could further explore 306 strategies to affect cognitive and social factors implicated in SMW's uptake of safer sex 307 practices. 
There are several study limitations. First, the cross-sectional design precludes making causal inferences between sexual stigma and safer sex practices. Second, an equal weight was applied to all practices in the Safer Sex Practices among Lesbian Women Scale. It should be noted that not all practices promote sexual safety equally, and a more nuanced analysis could

312 explore associations between stigma and specific practices. For example, are women with less 313 perceived stigma more sexually comfortable and more likely to discard less effective safe sex 314 practices? Third, while all included measures had adequate reliability and have been previously 315 used in studies with SMW, future studies may aim to study how these measures may be 316 improved. Fourth, measures were self-reported and social desirability bias may have influenced responses. Fifth, generalizability is limited due to the non-probability sampling strategy. Our

318 sample reflected predominantly white and highly educated participants, underrepresenting racial/ethnic minority women and those with less than university education. This could be due to the online survey method, biased to include people with internet access. ${ }^{45}$

322 apply minority stress theory to understand safer sex practices among SMW. Another strength is 323 our use of a comprehensive measure of safer sex sexual practices that accounts for the many 324 ways women have sex with women.

\section{Conclusions}

From a theoretical perspective, we found congruence with the minority stress model in

327 terms of social support and resilient coping as moderators in the relationship between stressors 328 targeting LGBT people and health outcomes. ${ }^{14}$ Yet we report complex relationships, whereby 329 social support level changed the nature of the association between enacted stigma and safer sex 330 practices, and resilient coping level altered the association between perceived stigma and safer 
331 sex practices. These findings challenge the current theoretical understanding that higher sexual

332 stigma is necessarily associated with lower uptake of safer sex strategies. Further research is

333 needed to empirically test theoretical reasons for these complex associations. For example, does

334 discrimination provide opportunities to solidify social support? If so, could there be a third

335 factor such as self-esteem that influences safer sex practices uptake? Are women who cope well

336 with perceived stigma more likely to notice stigma, cope well, and to practice safer sex? Future

337 studies should address intersectionality and the effect of multiple forms of stigma (e.g. racism)

338 on safer sex practices and the different needs of sexually diverse women (e.g. bisexual vs.

339 lesbian women). ${ }^{46}$

340 Our findings document complex relationships between sexual stigma dimensions, coping,

341 social support and safer sex practices that suggest the utility of the minority stress model for

342 understanding safer sex practices among SMW. This evidence can also inform safer sex

343 intervention development for SMW. 


\section{Acknowledgements}

346 This study was funded by the Canadian Institutes of Health Research (CIHR) Social Research

347 Centre in HIV Prevention. Grant number: CIHR: HCP-97106. The funders played no role in 348 study design, analysis or interpretation of results. Community partners include: Women's Health 349 in Women's Hands Community Health Centre, Black Coalition for AIDS Prevention, Planned 350 Parenthood Toronto, 519 Community Centre, Sherbourne Health Centre. Thank you to the peer 351 research assistants and participants.

352

353 Author Disclosure Statement

354 No competing financial interests exist. 


\section{References}

357 1. Estrich CG, Gratzer B, Hotton AL: Differences in sexual health, risk behaviors, and 358 substance use among women by sexual identity: Chicago, 2009-2011. Sex Transm Dis $359 \quad 2014 ; 41: 194-199$.

360 2. Bauer GR, Welles SL: Beyond assumptions of negligible risk: Sexually transmitted 361 diseases and women who have sex with women. Am J Public Health 2001;91:1282-1286.

362 3. Fethers K, Marks C, Mindel A, Estcourt CS: Sexually transmitted infections and risk 363 behaviours in women who have sex with women. Sex Transm Infect 2000;76:345-349.

364 4. Marrazzo JM: Sexually transmitted infections in women who have sex with women: Who 365 cares? Sex Transm Infect 2000;76:330-332.

366 5. Logie $\mathrm{CH}$, Gibson MF: A mark that is no mark? Queer women and violence in HIV 367 discourse. Cult Health Sex 2013;15:29-43.

368 6. Marrazzo JM, Gorgos LM: Emerging sexual health issues among women who have sex 369 with women. Curr Infect Dis Rep 2012 doi: 10.1007/s11908-012-0244-X

370 7. Marrazzo JM: Dangerous assumptions: Lesbians and sexual death. Sex Transm Dis $371 \quad 2005 ; 32: 570-571$.

372 8. Lenke K, Piehl M: Women who have sex with women in the global HIV pandemic. 373 Development 2009;52:91-94.

374 9. Pinto VM, Tancredi MV, Tancredi Neto A, Buchalla CM: Sexually transmitted 375 disease/HIV risk behaviour among women who have sex with women. AIDS 2005;19:64376 69. 
377 10. Schick V, Rosenberger JG, Herbenick D, Reece M: Sexual behaviour and risk reduction

378

379

380

381

382

383

384

385

386

387

388

389

390

391

392

393

394

395

396

397

398 399 strategies among a multinational sample of women who have sex with women. Sex Transm Infect 2012;88:407-412.

11. Baral S, Logie C, Grosso A, Wirtz AL, Beyrer C: Modified social ecological model: A tool to guide the assessment of the risks and risk contexts of HIV epidemics. BMC Public Health 2013;13:482.

12. Parkhurst JO: Structural approaches for prevention of sexually transmitted HIV in general populations: Definitions and an operational approach. J Int AIDS Soc 2014;17:19052.

13. Herek GM: Confronting sexual stigma and prejudice: Theory and practice. J Soc Issues 2007;63:905-925.

14. Meyer IH: Prejudice, social stress, and mental health in lesbian, gay, and bisexual populations: Conceptual issues and research evidence. Psychol Bull 2003;129:674-697.

15. Meyer IH: Minority stress and mental health in gay men. J Health Soc Behav $1995 ; 36: 38-56$.

16. Herek GM: Hate crimes and stigma-related experiences among sexual minority adults in the United States: Prevalence estimates from a national probability sample. J Interpers Violence 2009;24:54-74.

17. Hatzenbuehler ML: How does sexual minority stigma "get under the skin"? A psychological mediation framework. Psychol Bull 2009;135:707-730.

18. Diamant AL, Wold C: Sexual orientation and variation in physical and mental health status among women. J Womens Health 2003;12:41-49. 
400 19. Lea T, de Wit J, Reynolds R: Minority stress in lesbian, gay, and bisexual young adults in 401 Australia: Associations with psychological distress, suicidality, and substance use. Arch Sex Behav 2014;43:1571-1578.

403 20. Tebbe EA, Moradi B: Suicide risk in trans populations: An application of minority stress 404 theory. J Couns Psychol 2016. [e-pub ahead of print].

405 21. Swank E, Frost DM, Fahs B: Rural location and exposure to minority stress among 406 sexual minorities in the United States. Psychology \& Sexuality 2012;3:226-243.

407 22. Auerback JD, Parkhurst JO, Caceres CF: Addressing social drivers of HIV/AIDS for the 408 409 long-term response: conceptual and methodological considerations. Glob Public Health 2011;6:S293-S309.

410 23. Newcomb ME, Mustanski B: Moderators of the relationship between internalized 411 homophobia and risky sexual behavior in men who have sex with men: A meta-analysis.

413 24. Bontempo DE, D'Augelli AR: Effects of at-school victimization and sexual orientation on lesbian, gay, or bisexual youths' health risk behavior. J Adolesc Health 2002;30:364415 374.

416 25. Robinson JP, Espelage DL: Peer victimization and sexual risk differences between 417 lesbian, gay, bisexual, transgender, or questioning and nontransgender heterosexual 418 youths in grades 7-12. Am J Public Health 2013;103:1810-1819.

419 26. Arreola S, Santos GM, Beck J, et al.: Sexual stigma, criminalization, investment, and 420 access to HIV services among men who have sex with men worldwide. AIDS Behav 421 2015;19:227-234. 
422 27. Pearlin LI, Lieberman MA, Menaghan EG, Mullan JT: The Stress Process. J Health Soc $423 \quad$ Behav 1981;22:337-356.

424 28. Yi H, Sandfort TGM, Shidlo A: Effects of disengagement coping with HIV risk on 425 unprotected sex among HIV-negative gay men in New York City. Health Psychol $426 \quad 2010 ; 2: 205-214$.

427 29. Jeffries W, Marks G, Lauby J, et al.: Homophobia is associated with sexual behavior that 428

30. Corliss HL, Austin SB, Roberts AL, Molnar BE: Sexual risk in "mostly heterosexual" young women: Influence of social support and caregiver mental health. J Womens Health (Larchmt) 2009;18:2005-2010.

433 31. Young RM, Meyer IH. The trouble with "MSM" and "WSW": Erasure of the sexual434 435

33. Fishman SJ, Anderson EH: Perception of HIV and safer sexual behaviors among minority person in public health discourse. Am J Public Health 2005;95:1144-1149.

32. Magnani R, Sabin K, Saidel T, Heckathorn D: Review of sampling hard-to-reach and hidden populations for HIV surveillance. AIDS 2005;19:S67-S72. lesbians. J Assoc Nurses AIDS Care 2003;14:48-55.

34. Diaz RM, Ayala G, Bein E, et al.: The impact of homophobia, poverty, and racism on the mental health of gay and bisexual Latino men: Findings from 3 US cities. Am J Public Health 2001;91:927-932.

442 35. Logie $\mathrm{CH}$, Earnshaw V: Adapting and validating a scale to measure sexual stigma among 443 lesbian, bisexual and queer women. PLoS One 2015;10:e0116198. 
444 36. Sinclair VG, Wallston KA: The development and psychometric evaluation of the Brief

$445 \quad$ Resilient Coping Scale. Assessment 2004;11:94-101.

446 37. Zimet GD, Dahlem NW, Zimet SG, Farley GK: The multi-dimensional scale of perceived $447 \quad$ social support. J Pers Assess 1998;52:30-41.

448 38. Stata Statistical Software: Release 14 [computer program]. College Station, TX: $449 \quad$ StataCorp LP; 2015.

450 39. Frost DM, Meyer IH: Measuring community connectedness among diverse sexual

40. Aschbacher K, O'Donovan A, Wolkowitz OM, et al.: Good stress, bad stress and

42. Abdessamad HM, Yudin MH, Tarasoff LA, et al.: Attitudes and knowledge among obstetrician-gynecologists regarding lesbian patients and their health. J Womens Health

41. Richters J, Prestage G, Schneider K, Clayton S: Do women use dental dams? Safer sex practices of lesbians and other women who have sex with women. Sex Health 2010;7:165-169. (Larchmt) 2013;22:85-93.

43. Peel E, Thomson M: Editorial introduction: Lesbian, gay, bisexual, trans and queer health psychology: Historical development and future possibilities. Fem Psychol 2009;19:427436.

44. Logie C, Lacombe-Duncan A, Weaver J, et al.: An HIV and STI group-based intervention for lesbian, bisexual, queer and other women who have sex with women: 
466

467

468 45. Meyer IH, Wilson PA: Sampling lesbian, gay, and bisexual populations. Journal of

$469 \quad$ Counseling Psychology 2009;56:23-31.

470 46. McCauley HL, Silverman JG, Decker MR, et al.: Sexual and reproductive health

471 indicators and intimate partner violence victimization among female family planning

472

473

Results from a non-randomized cohort pilot study. AIDS Patient Care STDS $2015 ; 29: 321-328$.

clinic patients who have sex with women and men. J Womens Health (Larchmt)

$2015 ; 24: 621-628$. 
Table 1 Main socio-demographic characteristics of participants

\begin{tabular}{|c|c|c|}
\hline Variables $^{\mathrm{a}}$ & & No of participants (\%) \\
\hline Sexual Orientation & Queer & $175 \quad(45.5)$ \\
\hline \multirow[t]{5}{*}{$(n=385)$} & Lesbian & $116 \quad(30.1)$ \\
\hline & Bisexual & $62 \quad(16.1)$ \\
\hline & Gay & $17 \quad(4.4)$ \\
\hline & Other & $12(3.1)$ \\
\hline & Heterosexual & $3 \quad(0.8)$ \\
\hline \multirow[t]{9}{*}{ Ethno-racial identity* } & White/Caucasian & $256 \quad(66.0)$ \\
\hline & Black, African/Caribbean & $41 \quad(11.1)$ \\
\hline & Asian & $17 \quad(4.4)$ \\
\hline & South Asian & $15 \quad(3.9)$ \\
\hline & Indigenous/Aboriginal & $10 \quad(2.6)$ \\
\hline & Caribbean & $12 \quad(3.4)$ \\
\hline & Latina & $13 \quad(3.4)$ \\
\hline & Multiple ethnicities & $17 \quad(4.4)$ \\
\hline & Other ethnicities & $31 \quad(8.0)$ \\
\hline \multirow[t]{6}{*}{ Education } & Less than high school & $2 \quad(0.5)$ \\
\hline & High school or equivalent & $18 \quad(4.6)$ \\
\hline & Some college/university & $73 \quad(18.8)$ \\
\hline & College diploma & $48 \quad(12.4)$ \\
\hline & Bachelor degree & $144 \quad(37.1)$ \\
\hline & Graduate degree & $103 \quad(26.5)$ \\
\hline \multirow[t]{7}{*}{ Relationship status } & Dating (living together) & $125 \quad(32.2)$ \\
\hline & Dating (not living together) & 81 (20.9) \\
\hline & No current partners & $45 \quad(11.6)$ \\
\hline & Polyamorous/multiple partners & $58 \quad(15.0)$ \\
\hline & Married & $43 \quad(11.1)$ \\
\hline & Casual dating & $36 \quad(9.3)$ \\
\hline & & Mean $(S D)$ \\
\hline \multirow[t]{2}{*}{ Age $(n=350)$} & & $31.20 \quad(7.97)$ \\
\hline & & Median: 29.0 (Range: 18-70) \\
\hline \multirow[t]{2}{*}{ Annual income $(n=373)$} & & $33,679(25,648)$ \\
\hline & & $\begin{array}{l}\text { Median: 30,000 (Range: 0- } \\
175,000)\end{array}$ \\
\hline
\end{tabular}

${ }^{a} n=388$ unless otherwise specified

${ }^{*}$ Participants could select more than 1 response 
Table 2 Sexual practices and main outcome variables among participants

\begin{tabular}{|c|c|c|}
\hline Variables $^{a}$ & Mean (SD) & Number of participants (\%) \\
\hline $\begin{array}{l}\text { Healthcare utilization score } \\
(\mathrm{n}=384)\end{array}$ & $2.00(1.1)$ & \\
\hline Resilient coping $(n=386)$ & $15.9(2.8)$ & \\
\hline Social support $(n=378)$ & $47.6(8.9)$ & \\
\hline Perceived sexual stigma & $8.4 \quad(3.5)$ & \\
\hline Enacted sexual stigma $(n=383)$ & $3.5 \quad(2.7)$ & \\
\hline \multirow[t]{6}{*}{ Lifetime sexual partners } & $0-5$ & 74 (19.1) \\
\hline & $6-10$ & $99(25.5)$ \\
\hline & $11-15$ & $66(17.0)$ \\
\hline & $16-20$ & $37 \quad(9.5)$ \\
\hline & $21-50$ & $78 \quad(20.1)$ \\
\hline & $50+$ & $34 \quad(8.8)$ \\
\hline $\begin{array}{l}\text { Reported history of male } \\
\text { partner(s) }\end{array}$ & $\begin{array}{l}\text { Reported at least one } \\
\text { male partner }\end{array}$ & $121(31.2)$ \\
\hline \multirow{3}{*}{$\begin{array}{l}\text { Lifetime sexually transmitted } \\
\text { infection history }(n=363)\end{array}$} & Never had an STI & $284(78.2)$ \\
\hline & Had STI in the past & $59 \quad(16.3)$ \\
\hline & $\begin{array}{l}\text { Currently living with } \\
\text { STI }\end{array}$ & $20 \quad(5.5)$ \\
\hline
\end{tabular}

${ }^{a} n=388$ unless otherwise specified 


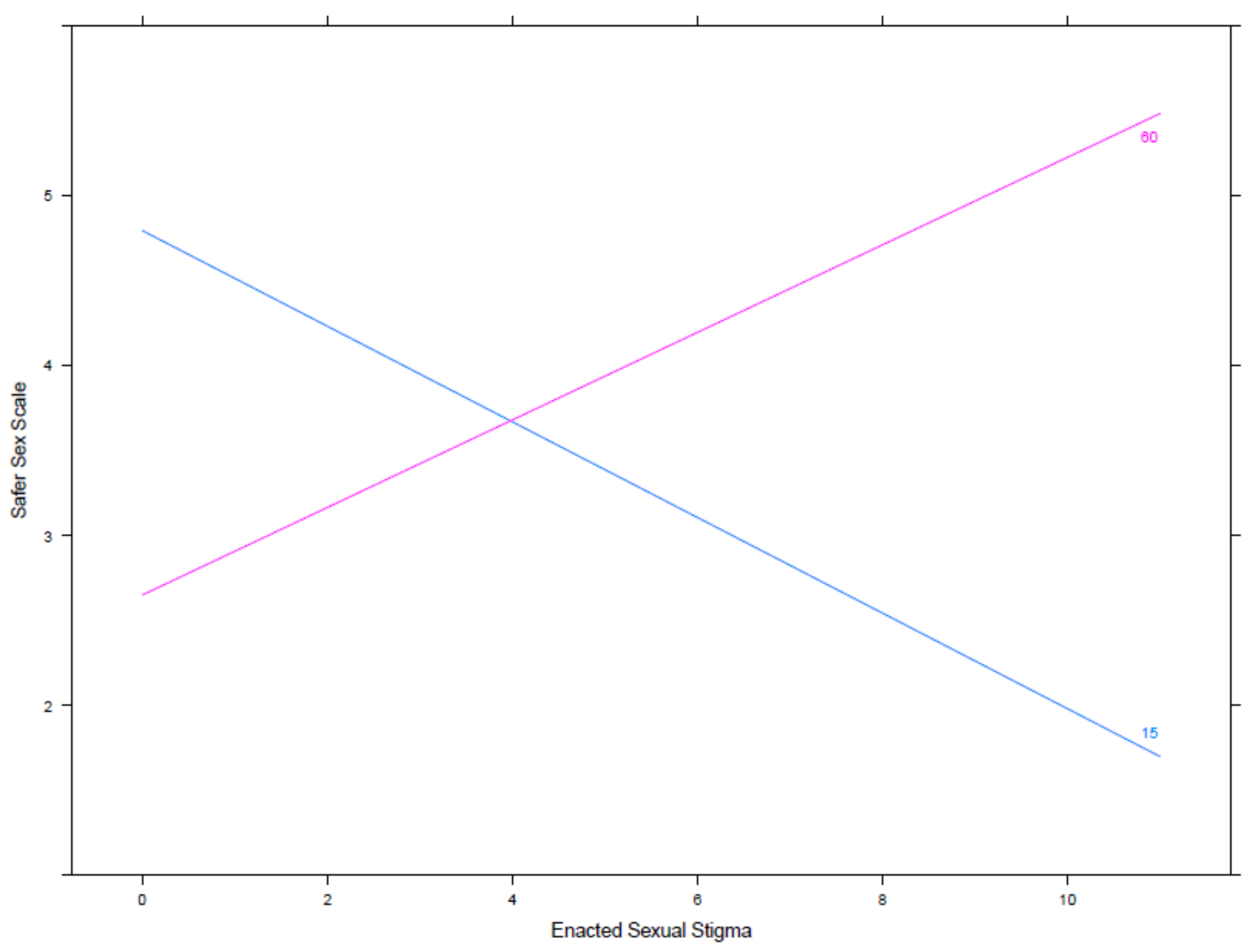

High Social Support Low Social Support 


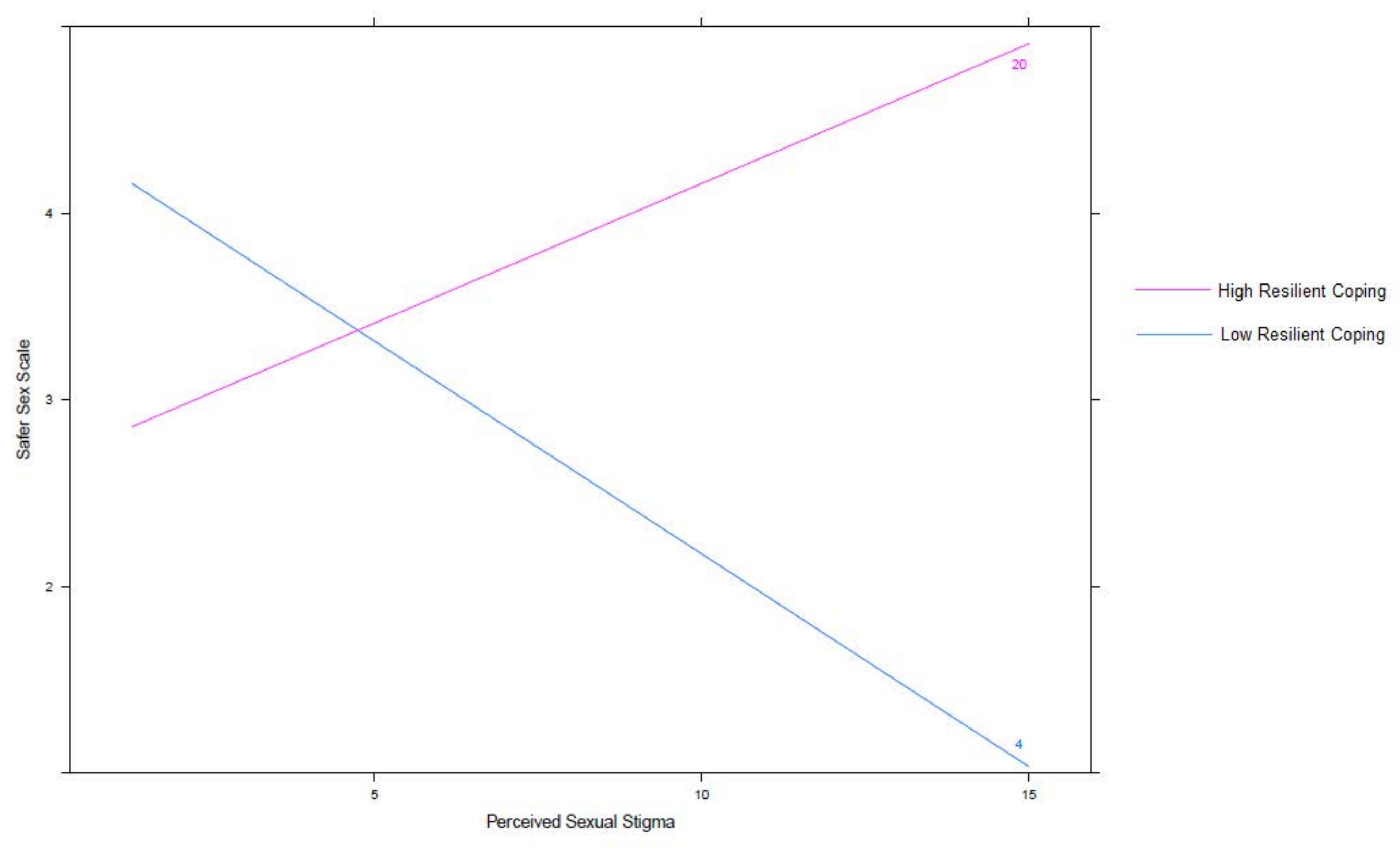

